\title{
2005 Association of American Physicians Presidential Address Academia-industry collaboration: A dynamic partnership on behalf of patients
}

Tadataka Yamada, M.D.

\begin{abstract}
L —adies and Gentleman, Colleagues, it is a great honor for me to address you today as the President of The Association of American Physicians. As I stand here, I am acutely aware of the storied 120-year history of our Association and humbled by the illustrious leaders of American medicine who have preceded me on this podium. In preparing for this day, I have had occasion to review the addresses of Presidents past and I asked myself what I could possibly add to the prescience and wisdom of the words already spoken. There are, of course, many topics of interest to me and, being an academic physician at heart, I am not without my opinions upon them. However, I have concluded that there is only one topic on which I might be somewhat uniquely qualified to comment from an informed point of view: the important interface between academia and industry.

Let me start by stating, after a 20 -year experience in academia and a 10-year experience in industry, that the underlying motive that drives the effort and commitment of most people in both camps is the desire to improve the lot of patients. In this light, a true partnership between industry and academia is both necessary and beneficial. To begin with, the two sides bring complementary capabilities and skill sets. If I am permitted a gross oversimplification, academia provides insight into the fundamental mechanisms of human disease and industry provides the knowledge and tools with which to convert these findings to practical application in patients. Academia provides expertise in patient care and clinical judgment while industry provides knowledge in clinical-trial design,
\end{abstract}

This article is adapted from a presentation at the ASCI/AAP Joint Meeting, April 15-17, 2005, in Chicago, Illinois, USA.

Address correspondence to: Tadataka Yamada, GlaxoSmithKline, Research and Development, 709 Swedeland Road, King of Prussia, Pennsylvania 19406, USA Phone: (610) 270-6300; Fax: (610) 270-5880; E-mail: tachi.yamada@gsk.com.

Citation for this article: J. Clin. Invest. 115:2944-2947 (2005). doi:10.1172/JCI26870.

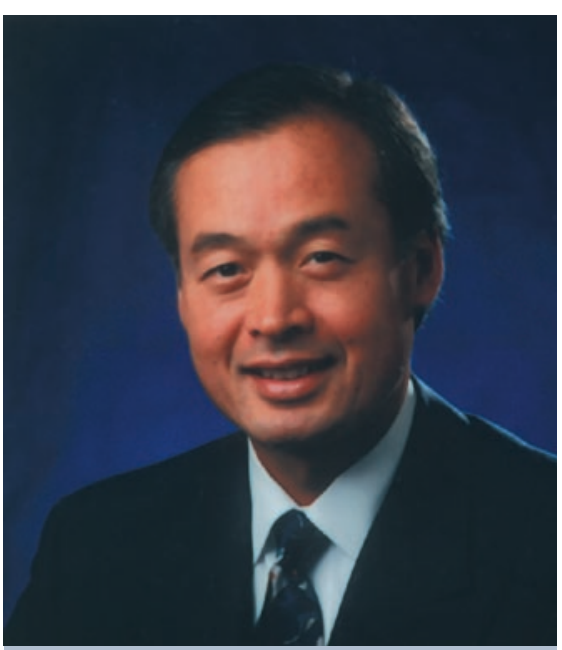

Tadataka Yamada, M.D., is Chairman, Research and Development, at GlaxoSmithKline and Adjunct Professor of Internal Medicine at the University of Michigan Medical School. He served as President of the Association of American Physicians from 2004 to 2005. He delivered his Presidential Address at the AAP's annual meeting, April 17, 2005. (Photograph courtesy of the author.)

data analysis and regulatory affairs. In reality the pharmaceutical industry is a fast-expanding major source of funding for research in academia and the principle source of funding for patient-oriented research. In return, industry gains valuable insight into its products from clinical experts in academia as well as pioneers in the basic sciences. Ultimately, of course, patients benefit from a better understanding of disease as well as better medicines to treat them.

In order to understand the underlying assumptions in academia-industry partnerships, it is important to explore the basis of the implied contract between the pharmaceutical industry and society. Industry assumes first that its mission is to apply the full weight of its technologies and resources to bring the best possible medicines to patients who need them. Because of the very nature of its products, industry accepts strict regulation of its activities in the interest of patient welfare. Implicit in the vital role that industry plays in biomedical research is a responsibility to share its innovations with the public in order to advance the cause of science and medicine. There is one important caveat: that this sharing must be commensurate with a viable competitive position. At times in the past, industry has considered that virtually everything it did to be competitive and proprietary. However, more recently, industry's view has shifted to believing that much if not most of the science it conducts is precompetitive in nature and increasingly the focus is on the chemical entities that form the drugs as the principle elements of intellectual property. Society, in regulating the pharmaceutical industry, makes a commitment to consider carefully both the benefits and the risks associated with new medicines. This is an increasingly important commitment to remember in the zerorisk-tolerance environment that is emerging in today's world. Furthermore, society has accepted that industry can expect to achieve a reasonable commercial return on its risky investments. This is viewed from an aggregate global perspective as industry is clearly also expected to provide medicines to the countries in the developing world with limited resources at a much reduced, if not negative, rate of return. An underlying assumption that enables industry to contemplate a commercial return on its investments given the high risk and prolonged timescale for drug development is that society will accept the notion of intellectual property or data protection as the bedrock of future innovations.

The making of new medicines is a difficult endeavor that requires the skills of many scientific perspectives. There are some in the industry who would begrudge the contributions of any academic laboratory in the evolution of their medicines. One need look no further than the largest class of medicines, the statins, and their derivation from the work of Goldstein and Brown, to refute such a preposterous notion. On the other hand, others would claim that the science of the 
pharmaceutical industry is strictly derivative and that the basis of all new drugs is the science that comes from academia. The storied history of GlaxoSmithKline with 5 Nobel laureates, Dale, Vane, Elion, Hitchings, and Black, would belie this notion. The truth is that a dynamic partnership between academia and industry has been the underpinning of almost all medicines. It is difficult to look closely at any medicine today and not see the intermingling of the work of molecular biologists, computational scientists, bioengineers, chemists, pharmacologists, pharmaceutical technologists, clinicians, and statisticians from both academia and industry.

Over the years, the relationships between academia and industry have taken many shapes. In its most basic form, industry recognizes the unique ability of academia to explore the fundamental basis of human disease. Therefore it seeks access to the intellectual property housed within universities and enabled by the Bayh-Dole Act of 1980, which literally freed academic institutions to express their own entrepreneurial instincts. Generally these relationships involve straightforward negotiations leading to contracts consisting of up-front payments, milestones, and royalties. Such agreements are mutually beneficial, with industry obtaining access to key technologies and academics seeing their basic discoveries applied to practice. There is a cloud on the horizon, however; the royalties resulting from these contracts can add up to a substantial proportion of the commercial potential of certain products, biopharmaceuticals in particular. The net result could be either a decision not to proceed with a program or the initiation of an effort to work around the blocking intellectual property, which may be time-consuming and costly. Neither result would be in the best interest of patients.

A much more natural relationship between industry and academia is that between individual scientists. As industry has come to understand that not everything it does is proprietary and requiring of protection, it has become far easier to develop scientist-to-scientist interactions for mutual benefit. In addition to collaboration at a laboratory scale, there is an emerging recognition that big scientific problems require large collaborative interactions between government, universities, funding agencies, and industry. A model example of such collaboration was the SNP Consortium, which provided the world with an incomparable database for application to future biomedical research. The notion that such collaborations are "pre-competitive" has allowed the industry to participate in them with increasing frequency, with the result that such efforts will decrease wasteful duplication of effort, increase productivity, and drive down the costs of pharmaceutical discovery. Today there are active discussions about publicprivate efforts in the areas of biomarkers and surrogates, structural genomics, mouse knockouts, and population cohorts, just to name a few.

One of the most exciting consequences of the power of genomics is the enormous number of new targets for drug action that have been disclosed. Testing and validation of these targets have proved to be a challenge, but increasingly the most appropriate experimental model for analysis is the human. In the 21 st century, experimental medicine has emerged as the science of the day, recognizing the human as the preferred model organism for biomedical research. In this discipline, physician-scientists are the keys to success because of their ability to cross the divide between basic science and clinical medicine. Industry desperately needs the collaboration with academia in experimental medicine. Early studies in humans that are well-conducted, meaningful, and valid not only help to validate drug targets but also offer insights that lead to better clinical protocols and avoidance of costly mistakes in larger-scale patient trials. The big challenge is that at the same time demand is soaring for physician-scientists who are uniquely qualified to excel in this emerging scientific discipline, the supply is dwindling and alarmingly endangered. The future vitality of both academic medicine and pharmaceutical development are dependent upon meeting this challenge effectively. Accordingly, a true partnership between academia and industry is necessary for the planning and implementation of solutions to this crisis.

The bread and butter of the pharmaceutical industry are large-scale registration studies. It is on the basis of so-called Phase II and Phase III studies in thousands of patients that medicines are approved and registered. These efforts are highly regulated in all their stages by government authorities. Generally the protocols are derived by industry but only after careful and extensive consultation with academic experts in the disease area and with regulatory authorities. For these studies, the data must be held centrally and monitored by all stakeholders. In the end no element of these studies can be withheld; indeed, complete disclosure of the entire dataset from these studies to the authorities is an absolute requirement. A major challenge to academics engaged in these studies is the requirement for rigid adherence to the protocols and fastidious record keeping. Following the registration of products, regulatory authorities often mandate one or more additional studies, generally called Phase IV studies. They are meant to answer questions that have arisen from or were not adequately addressed in the pivotal pre-registration programs. Sometimes they are meant to address safety issues but often they are landmark studies which can change the practice of medicine. The $4 \mathrm{~S}$ studies come to mind. These studies firmly established that lowering LDL cholesterol levels in serum can result in reduced mortality from cardiovascular events. Largescale clinical trials, including Phases II-IV, represent unique opportunities to ask and answer medical questions of great importance. As their costs are huge, they are meticulously planned with the full participation of academic experts who are vital to their successful implementation. These trials often have wide public visibility because of their potential impact on medical practice. In this light, it is difficult to understand why the academic participants in such studies seldom receive appropriate career recognition for their efforts.

In addition to the clinical trials that are driven from the drug registration process, there are two other types of studies that are worthy of mention. The first, investigator-initiated studies, usually results from a concurrence of interest between academic and industry partners. For many clinician scholars, this is a vital means of support for maintaining the scientific thrust of their research. At their best, these studies can provide novel insights into medicines, biological pathways, and disease states. Few other avenues of support are available for clinical investigators to explore legitimate scientific questions in humans. At their worst, however, they can provide vehicles by which academic physicians can be rewarded in a surreptitious fashion by companies for the support of a product in the marketplace. There is risk for industry in these studies as well; sometimes the studies can be poorly conducted and jeopardize a product's label. In addition they may produce results which are most 
unfavorable for the sponsoring industry partner. The conduct of investigator-initiated studies clearly requires a transparent contract between the academic and the pharmaceutical company in which both parties fully acknowledge and accept the risks involved. The second type of non-registration-focused studies are the so-called marketing studies. By their very nature they are intended for the purpose of enhancing the commercial profile of a product. They are usually limited in size and scope. Often they are not statistically powered to derive a firm conclusion, thus the results frequently do not influence product labeling. For investigators involved in these studies there is a high risk of conflict of interest as well as undue pressure for results to come out a certain way. Before the recent commitment by industry to publish more freely results of all clinical trials, these studies were most likely to remain undisclosed if the results were not favorable. Although marketing studies constitute only the smallest fraction of clinical trials conducted, they have undoubtedly created the largest fraction of issues that have arisen at the academicindustrial interface.

From the discussion of various relationships between pharmaceutical companies and academic scientists, it should be obvious that most are honestly motivated, highly productive, mutually beneficial, and supportive of the best interests of patient care. Nevertheless there are many thorny issues affecting the relationship. They fall into four categories: integrity of research, ownership of data, publication and disclosure, and conflicts of interest. It is worthwhile to consider each carefully.

Nothing strikes at the core of the reputation of biomedical research so much as the question of integrity of research. Regrettably, there are very public examples from both industry and academia which re-inforce an image of scientists cheating at the expense of the public. However, an often overlooked reality of the pharmaceutical industry is that it is one of the most highly regulated of all. In the United States, the Food and Drug Administration requires the industry to abide by a series of principles called GLP (Good Laboratory Practice), GCP (Good Clinical Practice) and GMP (Good Manufacturing Practice). The consequences of non-adherence to these principles can be disapproval of protocols, termination of ongoing studies, rejection of a registration file, revocation of a license, and even seizure of products in the market- place. While there is no exact counterpart in the academic world for such regulatory requirements, there are increasing pressures from institutional review boards, university ethics panels, and even Congress to adhere to strict standards of integrity. For any academic-industry research collaboration there are a few elements of technical integrity to keep in mind. First and foremost, there must be an assurance that there will be no inappropriate influence on data selection or interpretation. Secondly, there should be assurance of technical compliance and quality control; clearly the studies must apply valid experimental methods. Lastly, there should be full access to data and participation in its analysis and presentation. Following these principles will keep academic-industry collaborations on the highest plane.

The ownership of data collected in industry-sponsored clinical trials has been the subject of substantial debate. Investigators who engage in clinical research understandably feel that the data they collect are rightfully owned by them for use toward an academic purpose. Major scientific problems occasionally arise when investigators wish to analyze and publish the results obtained from a subset of a data pool, as for example from one site or country in a multi-center international clinical trial, the results of which may differ from the results of the complete trial. Obviously the integrity of the trial cannot be maintained unless the results of the trial are analyzed with the data from the entirety of the enrolled patient cohort. The regulations that apply to medicines that have not yet been registered are explicit and clear. The guidelines for Good Clinical Practice under the rules governing the pharmaceutical industry (ICH-6, to which all major regulatory authorities adhere) require that the study sponsor take responsibility for data recording, reporting, quality assurance, analysis and regulatory review, and for retaining these data. The intent, of course, is for protection of trial subjects because unless all the data are retained and maintained under one accountable authority, safety and pharmacovigilance cannot be maintained. On the other hand, the rules may be quite different for studies that are not sponsored by pharmaceutical companies and which take place after products are available in the market. It would seem unreasonable for pharmaceutical companies to claim data ownership in investigator-initiated trials of registered products even if they have agreed to sponsor them, unless there is an explicit agreement to that effect prior to the initiation of the research. Ultimately, the issue of data ownership is moot; the real issue is the rigor and integrity with which the data are analyzed and the results reported.

There is growing concern in the general public that selective reporting of clinical trial results has led to under-reporting of negative data and perhaps even closeting of critical safety information. Two recent examples that have fuelled some of this concern are the risks of pediatric suicidality associated with anti-depressants and cardiovascular mortality associated with COX-2 inhibitors. Major initiatives pushing for more complete disclosure of the results of clinical trials have come from journal editors, state attorneys general, and from the United States Congress. Responding to the growing public demand for greater transparency, the Pharmaceutical Research and Manufacturers Association (PhRMA) issued a document titled "Principles on the Conduct of Clinical Trials and Communication of Clinical Trial Results," which commits the pharmaceutical industry to "timely communication of meaningful results of controlled clinical trials of marketed products or investigational products that are approved for marketing, regardless of outcome. Communication includes publication of a paper in a peerreviewed medical journal, abstract submission with a poster or oral presentation at a scientific meeting, or making results public by some other means." Although these principles include a "carve-out" for exploratory studies that might be proprietary in nature, some companies have established an independent policy committing to disclose the results of all studies conducted in patients. Because of the requirement for timely disclosure, individual companies as well as PhRMA have established websites through which the results of studies could be disclosed if publications were unavailable or not forthcoming. A newer wrinkle to this issue is the request by journal editors that protocols for clinical trials be disclosed prior to initiating them. This request is driven by a conviction that for proper review of manuscripts on the results of clinical trials, full transparency of their original design, construct, and endpoints is required. Because of the proprietary and competitive nature of some of these trials, many pharmaceutical companies have expressed reluctance to commit to such a policy. Nevertheless, PhRMA has brokered 
an agreement among its members to place protocols for clinical trials in a secured website prior to initiation of studies and to allow these protocols to be accessed in a confidential manner only by journal editors or manuscript reviewers when papers are submitted for publication. All of the developments within the past year indicate an increasing awareness on the part of the pharmaceutical industry of its responsibility to be transparent with the important clinical data in their possession. What remains uncharted territory however is the willingness and the ability of academic scientists to adhere to these same principles.

In any relationship of an academic scientist with a for-profit commercial entity such as a pharmaceutical company, there are inevitable questions of potential misconduct arising from conflicts of interest. The potential for misconduct is assumed to derive from a profit motive, but this assumption is debatable. When one examines the history of highly publicized cases of scientific misconduct, there are certainly examples in which industry has been involved but perhaps equally well-known are examples involving no industry partner, just misguided individuals seeking fame, advancement, or other recognition. Indeed, the environment of tight regulatory control governing pharmaceutical research makes scientific misconduct difficult at the very least. If medicines are ineffective or unsafe, they will not survive in the marketplace. From a purely commercial perspective, pharmaceutical companies have a fiduciary responsibility to their shareholders, and scientific misconduct leading to bad outcomes or withdrawal of products from the market can have catastrophic consequences on shareholder value. Since the end prod- ucts of pharmaceutical research are medicines that are used by patients, to whom we are ultimately all accountable, there is the inevitable court of clinical outcome that tests results of clinical trials. Some 15 years ago, I had the honor of chairing a committee that drafted guidelines for the responsible conduct of research at the University of Michigan Medical School. In it, I remember writing one paragraph that stands out as being applicable to industry and academia alike: "The extensive system of peer review that begins in our own laboratories or institutions, and intensifies upon application of grant funding or following submission of a manuscript for publication, limits the viability of a biomedical scientist who does not adhere strictly to open and honest practices. In the final analysis, the veracity of a work of a biomedical scientist is judged by the test of time."

In order to prevent conflicts of interest from arising in relationships between industry and academia, there are a few principles to consider. From a financial standpoint all arrangements should be transparent, there should be clear demarcation of the contributions and rewards, and there should be clear audit trails and independent verification of the arrangements. From the standpoint of personal integrity, there should be adherence to sound scientific and ethical principles, respect for intellectual property, and fair and appropriate allocation of effort as well as distribution of credit. Adhering to these principles would contribute greatly to maintaining a healthy relationship between academia and industry.

Looking to the future, it is difficult for me to envision anything but greatly enhanced collaborations between academia and indus- try. All of us are engaged in the endeavor of patient care, and as medicines are the ultimate translation of basic biomedical science into clinical practice, we owe it to our patients to build effective academiaindustry collaborations. One of the exciting developments in the recent past has been the increasing ease with which academics have found their way into industry. While the way back from industry to academia is yet only a narrow path, it is growing wider by the day. This increasingly flexible interchange of human capital and skills is necessary and important for the optimization of the collaboration between industry and academia. A gratifying reality is that collaborations are definitely on the rise and they are becoming significant from a scientific standpoint. There is not a week that goes by when I am not engaged in dialogue with the NIH, the Wellcome Trust, the UK Medical Research Council, or a university to discuss a project with profound consequences in the science of medicine. From biomarkers to the training of physician-scientists, from largescale patient cohorts to a mouse knock-out consortium, from an alternative drug-discovery initiative to a cancer clinical-trial network, public-private collaborations are the keys to success. There are issues at the interface, but none are insoluble. Mutual respect and understanding will win the day. In the end, we have a common global mission: to improve the quality of life for all of our patients.

Let me close this address by expressing my deepest gratitude for the opportunity to serve as the President of the Association of American Physicians. It has been a profound honor to serve you who comprise the membership of our august association. Thank you. 